PROCEEDINGS OF THE

AMERICAN MATHEMATICAL SOCIETY

Volume 134, Number 8 , Pages $2335-2347$

S 0002-9939(06)08277-3

Article electronically published on February 6, 2006

\title{
SYMMETRIZATION INEQUALITIES AND SOBOLEV EMBEDDINGS
}

\author{
JOAQUIM MARTÍN AND MARIO MILMAN
}

(Communicated by Andreas Seeger)

\begin{abstract}
We prove new extended forms of the Pólya-Szegö symmetrization principle. As a consequence new sharp embedding theorems for generalized Besov spaces are proved, including a sharpening of the limiting cases of the classical Sobolev embedding theorem. In particular, a surprising self-improving property of certain Sobolev embeddings is uncovered.
\end{abstract}

\section{INTRODUCTION}

The Pólya-Szegö symmetrization principle and its variants play a fundamental role in many problems in analysis. The classical version of the Pólya-Szegö principle states that

$$
\left\|\nabla f^{\circ}\right\|_{L^{p}\left(\mathbb{R}^{n}\right)} \leq\|\nabla f\|_{L^{p}\left(\mathbb{R}^{n}\right)},
$$

where $f^{\circ}$ denotes the symmetric rearrangement of $f \in W_{1}^{1}\left(\mathbb{R}^{n}\right)$ (cf. 24], [1]). More generally, in 8 it is shown that

$$
\left|\nabla f^{\circ}\right|^{* *}(t) \leq|\nabla f|^{* *}(t),
$$

where $f^{* *}(t)=\frac{1}{t} \int_{0}^{t} f^{*}(s) d s$, and $f^{*}$ denotes the non-increasing rearrangement of $f \in C_{0}^{\infty}\left(\mathbb{R}^{n}\right)$. It follows from (1.2) that for any rearrangement invariant space (r.i. space) $X\left(\mathbb{R}^{n}\right)$ we have (cf. also [15])

$$
\left\|\nabla f^{\circ}\right\|_{X\left(\mathbb{R}^{n}\right)} \leq\|\nabla f\|_{X\left(\mathbb{R}^{n}\right)}, f \in C_{0}^{\infty}\left(\mathbb{R}^{n}\right) .
$$

A natural way to measure the oscillation of a decreasing function is provided by the quantity (cf. [3])

$$
f_{o}^{*}(t)=f^{* *}(t)-f^{*}(t) .
$$

Using (1.2) the following inequality for $f_{o}^{*}(t)$ was shown in [2]:

$$
f_{o}^{*}(t) \leq c_{n} t^{1 / n}|\nabla f|^{* *}(t), f \in C_{0}^{\infty}\left(\mathbb{R}^{n}\right) .
$$

Received by the editors August 25, 2004 and, in revised form, March 8, 2005.

2000 Mathematics Subject Classification. Primary 46E30, 26D10.

Key words and phrases. Symmetrization, Besov spaces, Sobolev spaces, rearrangement invariant spaces.

The first author was supported by "programa Ramón y Cajal (MCYT)", and in part by MTM2004-02299 and CURE 2001SGR 00069.

${ }^{1}$ A different approach to a result involving $f^{*}(t)-f^{*}(2 t)$ had been given earlier in [17. Note (cf. [2]) that we always have $f^{*}(t)-f^{*}(2 t) \leq 2 f_{o}^{*}(t), t>0$.

(C)2006 American Mathematical Society Reverts to public domain 28 years from publication 
In [2, 23] it is shown that (1.4) implies the following improvement to the Hansson-Brézis-Wainger-Maz'ya 2 limiting version of the Sobolev embedding theorem:

$$
\stackrel{\circ}{\mathrm{W}}_{n / k}^{k}(\Omega) \subset L\left(\infty, \frac{n}{k}\right)(\Omega) \varsubsetneqq H_{\frac{n}{k}}(\Omega),
$$

where $\Omega$ is a bounded domain in $\mathbb{R}^{n}$ and $\stackrel{\circ}{W}_{n / k}^{k}(\Omega)$ denotes the closure of $C_{0}^{\infty}(\Omega)$ in the norm $\|f\|_{\stackrel{\mathrm{W}}{n / k}_{n}^{k}(\Omega)}=\left\|\nabla^{k} f\right\|_{L^{n / k}(\Omega)}$.

In this note we consider the fractional case. In fact we shall obtain rearrangement inequalities for moduli of continuity which allow us to find a suitable replacement for (1.4) in the fractional case, and derive applications to embeddings of Besov spaces.

Recall the analogue of (1.1) for $L^{p}$ moduli of continuity (cf. [10, 9] and the references therein):

$$
\omega_{p}\left(t, f^{\circ}\right) \leq c \omega_{p}(t, f) .
$$

A version of (1.6) for Orlicz spaces was given in [10], 9], with a complicated proof, and one can find a version of (1.6) valid for r.i. spaces in dimension one in 22 .

Our first result (see Section 3. Theorem 1 below) is a generalization of (1.6) for r.i. spaces $X\left(\mathbb{R}^{n}\right)$ :

$$
\omega_{X}\left(t, f^{\circ}\right) \leq c \omega_{X}(t, f),
$$

where $\omega_{X}:(0, \infty) \times X\left(\mathbb{R}^{n}\right) \rightarrow[0, \infty)$ is the $X\left(\mathbb{R}^{n}\right)$ modulus of continuity defined by

$$
\omega_{X}(t, g)=\sup _{|h| \leq t}\|g(.+h)-g(.)\|_{X} .
$$

It is of interest that our method of proof of (1.7), which is based on real interpolation, simplifies considerably the combinatorial methods of Garsia-Rodemich [10], where the case of Orlicz spaces is considered. Using (1.7) we can estimate the oscillation $f_{o}^{*}(t)$, assuming a priori that $f$ belongs to a r.i. space $X\left(\mathbb{R}^{n}\right)$ (see Theorem 2 below):

$$
f_{o}^{*}(t) \leq c \frac{\omega_{X}\left(t^{1 / n}, f\right)}{\phi_{X}(t)}
$$

where $\phi_{X}(t)$ denotes the fundamental function 3 of $X\left(\mathbb{R}^{n}\right)$. For an $L^{p}\left([0,1]^{n}\right)$ version of (1.9) see [16], 17] and the references quoted therein.

In Section 4 we use (1.9) to derive sharp embedding theorems for generalized Besov spaces. In particular, we are able to sharpen known limiting embedding theorems for classical Besov spaces (based on $L^{p}$ spaces) as follows: let $1 \leq q \leq \infty, 0<\alpha<1$; then if we let $\stackrel{\circ}{\mathrm{B}}_{n / \alpha, q}^{\alpha}\left(\mathbb{R}^{n}\right)$ be the closure of $C_{0}^{\infty}\left(\mathbb{R}^{n}\right)$ under $\left(\int_{0}^{\infty}\left(t^{-\alpha} \omega_{L^{n / \alpha}}(t, f)\right)^{q} \frac{d t}{t}\right)^{1 / q}$, we have

$$
\stackrel{\circ}{\mathrm{B}}_{n / \alpha, q}^{\alpha}\left(\mathbb{R}^{n}\right) \subset L(\infty, q)\left(\mathbb{R}^{n}\right)
$$

\footnotetext{
${ }^{2}$ Here $L\left(\infty, \frac{n}{k}\right)(\Omega)=\left\{f:\|f\|_{L\left(\infty, \frac{n}{k}\right)}^{n / k}=\int_{0}^{|\Omega|} f_{o}^{*}(s)^{n / k} \frac{d s}{s}<\infty\right\}$, and the Hansson-BrezisWainger space (cf. [1], [5]) is defined by $H_{n / k}(\Omega)=\left\{f:\|f\|_{\left.H_{n / k} / k\right)}^{n / k}=\int_{0}^{|\Omega|}\left(\frac{f^{* *}(s)}{\left(1+\ln \frac{|\Omega|}{s}\right)}\right)^{n / k} \frac{d s}{s}<\right.$ $\infty$ \}. We should note that the embedding $\stackrel{\circ}{\mathrm{W}}_{n}^{1}(\Omega) \subset H_{n}(\Omega)$ can also be obtained using some fundamental capacitary inequalities due to Maz'ya [21] .

${ }^{3}$ See Section 2 below.
} 
This extend4 4 the Herz-Peetre Sobolev embedding (cf. [13, 25]) to the limiting case $n / \alpha$. The proof of (1.10) can be obtained by means of applying (1.9) with $X=L^{n / \alpha}$, for then we have

$$
f_{o}^{*}(t) \leq c \frac{\omega_{L^{n / \alpha}}\left(t^{1 / n}, f\right)}{t^{\alpha / n}}
$$

Therefore,

$$
\begin{aligned}
\int_{0}^{\infty} f_{o}^{*}(t)^{q} \frac{d t}{t} & \leq c \int_{0}^{\infty}\left(t^{-\alpha} \omega_{L^{n / \alpha}}(t, f)\right)^{q} \frac{d t}{t} \\
& =c\|f\|_{\dot{\mathrm{B}}_{n / \alpha, q}^{\alpha}}^{q},
\end{aligned}
$$

and (1.10) follows. It is interesting to stress here that the general form of (1.9) allows us to extend (1.10) as follows: let $X=L\left(\frac{n}{\alpha}, \infty\right)$; then $\phi_{L\left(\frac{n}{\alpha}, \infty\right)}(t)=t^{\alpha / n}$, and the proof given above self improves to

$$
\stackrel{\circ}{\mathrm{B}}_{n / \alpha, q}^{\alpha}\left(\mathbb{R}^{n}\right) \subset \stackrel{\circ}{\mathrm{B}}_{L\left(\frac{n}{\alpha}, \infty\right), q}^{\alpha}\left(\mathbb{R}^{n}\right) \subset L(\infty, q)\left(\mathbb{R}^{n}\right) .
$$

More generally, all the Sobolev-type embeddings we prove in this note have the following apparently new self-improving property 5 if $X, Y, Z$ are r.i. spaces, then

$$
\stackrel{\circ}{\mathrm{B}}_{X, Y}^{\alpha} \subset Z \Rightarrow \stackrel{\circ}{\mathrm{B}}_{M(X), Y}^{\alpha} \subset Z,
$$

where $M(X)=\left\{f:\|f\|_{M(X)}=\sup _{t>0}\left\{f^{*}(t) \phi_{X}(t)\right\}<\infty\right\}$ is the Marcinkiewicz space associated with $X$ (note that $\left.\phi_{M(X)}(t)=\phi_{X}(t)\right)$.

Using (1.10) and the known embedding theorems of potential spaces in Besov spaces [27, Theorem $5(\mathrm{~A})$, page 155] we obtain 6

$$
\dot{\mathcal{L}}_{\alpha}^{n / \alpha}\left(\mathbb{R}^{n}\right) \subset L\left(\infty, \frac{n}{\alpha}\right)\left(\mathbb{R}^{n}\right), n \geq 2 \alpha .
$$

If we observe that $\frac{d}{d t} f^{* *}(t)=-t^{-1} f_{o}^{*}(t)$ we see that (1.9) implies the following extension to r.i. spaces of a result in [4, Theorem 4.19, page 345] (see also [10] for related results obtained in the context of Orlicz spaces):

$$
f^{* *}(t) \leq c \int_{t^{1 / n}}^{\infty} \frac{\omega_{X}(s, f)}{\phi_{X}\left(s^{n}\right)} \frac{d s}{s}, \text { if } f^{*}(\infty)=0 .
$$

\section{Preliminaries}

2.1. Background on rearrangement invariant spaces. Let $X\left(\mathbb{R}^{n}\right)$ be a r.i. space. Recall that $X=X\left(\mathbb{R}^{n}\right)$ has a representation as a function space on $(0, \infty)$, $X^{\wedge}(0, \infty)$ such that (cf. [4])

$$
\|f\|_{X\left(\mathbb{R}^{n}\right)}=\left\|f^{*}\right\|_{X^{\wedge}(0, \infty)},
$$

where $f^{*}$ denotes the non-increasing rearrangement defined by

$$
f^{*}(t)=\inf \left\{\lambda>0: \operatorname{mes}\left\{x \in \mathbb{R}^{n}:|f(x)|>\lambda\right\} \leq t\right\} .
$$

The symmetric spherical decreasing rearrangement $f^{\circ}$ is defined by

$$
f^{\circ}(x)=f^{*}\left(c_{n}|x|^{n}\right), \quad x \in \mathbb{R}^{n},
$$

\footnotetext{
${ }^{4}$ The Herz-Peetre embedding also follows directly from (1.9).

${ }^{5}$ This self-improving property is reminiscent of the self-improving properties of the method of truncation due to Maz'ya (cf. [20], 12]).

${ }^{6}$ This partially extends (1.5) to potential spaces. For other limiting cases of the Sobolev embedding theorem for potential spaces see [11], [5], 7].
} 
where $c_{n}=$ measure of the unit ball in $\mathbb{R}^{n}$. It is plain that $f^{\circ}$ is equimeasurable with $f$, and therefore

$$
\|f\|_{X\left(\mathbb{R}^{n}\right)}=\left\|f^{\circ}\right\|_{X^{-}\left(\mathbb{R}^{n}\right)} .
$$

We refer the reader to [4], [18] and [24] for further information on non-increasing rearrangements and symmetric spherical rearrangements. Moreover, since the ambient domain will always be clear from the context it is convenient to "drop the hats and tildes" and use the same letter $X$ to indicate the different versions of the space $X$ that we use. Moreover, since it will simplify our considerations we shall assume in what follows that $C_{0}^{\infty}\left(\mathbb{R}^{n}\right)$ is dense in $X\left(\mathbb{R}^{n}\right)$.

Let $\stackrel{\circ}{W_{X}^{1}}=\stackrel{\circ}{W_{X}^{1}}\left(\mathbb{R}^{n}\right)=\{f \in X: \nabla f \in X\}$. We equip $\stackrel{\circ}{W_{X}^{1}}$ with the seminorm

$$
\|f\|_{W_{X}^{1}}^{\circ}=\|\nabla f\|_{X}
$$

It follows (cf. [4], 14] )) that the $K$-functional for the pair $\left(X, \stackrel{\circ}{W_{X}^{1}}\right)$ defined by

$$
K\left(t, f ; X, \stackrel{\circ}{W_{X}^{1}}\right)=\inf _{g \in W_{X}^{1}}\left\{\|f-g\|_{X}+t\|\nabla g\|_{X}\right\}
$$

is equivalent to the $X\left(\mathbb{R}^{n}\right)$-modulus of continuity (cf. (1.8) above):

$$
K\left(t, f ; X, \stackrel{\circ}{W_{X}^{1}}\right) \simeq \omega_{X}(t, f),
$$

where as usual, the symbol $f \simeq g$ will indicate the existence of a universal constant $c>0$ (independent of all parameters involved) so that $(1 / c) f \leq g \leq c f$, while the symbol $f \preceq g$ means that $f \leq c g$.

2.1.1. Indices. Let $D_{\frac{1}{s}} f(t)=f\left(\frac{t}{s}\right), s>0$, be the dilation operator, and let $h_{X}(s)$ be the norm of the dilation operator, i.e.,

$$
h_{X}(s)=\sup _{f \in X} \frac{\left\|D_{\frac{1}{s}} f\right\|_{X}}{\|f\|_{X}}, s>0 .
$$

The upper and lower Boyd indices associated with a r.i. space $X$ are defined by

$$
\bar{\alpha}_{X}=\inf _{s>1} \frac{\ln h_{X}(s)}{\ln s} \text { and } \underline{\alpha}_{X}=\sup _{s<1} \frac{\ln h_{X}(s)}{\ln s} .
$$

It is also useful sometimes to consider a slightly different set of indices obtained by means of replacing $h_{X}(s)$ in (2.1) by

$$
M_{X}(s)=\sup _{t>0} \frac{\phi_{X}(t s)}{\phi_{X}(t)}, s>0 .
$$

The corresponding indices are denoted $\bar{\beta}_{X}, \underline{\beta}_{X}$, and will be referred to as the upper and lower fundamental indices of $X$. Actually, the relationship between $M_{X}(s)$ and $h_{X}(s)$ is that the computation of the former is exactly the computation of the latter but done only over functions of the form $f=\chi_{(0, a)}$. Therefore we have (cf. [4])

$$
0 \leq \underline{\alpha}_{X} \leq \underline{\beta}_{X} \leq \bar{\beta}_{X} \leq \bar{\alpha}_{X} \leq 1 \text {. }
$$

\footnotetext{
${ }^{7} \phi_{X}(s)$ is the fundamental function of $X: \phi_{X}(s)=\left\|\chi_{E}\right\|_{X}$, with $E$ any measurable subset of $\Omega$ with $|E|=s$.
} 
We shall usually formulate conditions on r.i spaces in terms of the Hardy operators defined by

$$
P f(t)=\frac{1}{t} \int_{0}^{t} f(x) d x ; \quad Q f(t)=\int_{t}^{\infty} f(x) \frac{d x}{x} .
$$

In particular, it is well known (cf. [4]) that if $X$ is a r.i. space, $P: X(0, \infty) \rightarrow$ $X(0, \infty)($ resp. $Q)$ is bounded if and only if $\bar{\alpha}_{X}<1$ (resp. $0<\underline{\alpha}_{X}$ ).

2.1.2. Some new function spaces. Following [23] and 26] we shall now construct the range spaces for our generalized Sobolev embedding theorem. Suppose that $X$ and $Y$ are r.i. spaces, and let $s \in \mathbb{R}$. We define

$$
Y(\infty, s, X)=\left\{f:\|f\|_{Y(\infty, s, X)}=\left\|t^{-s} \frac{\phi_{X}(t)}{\phi_{Y}(t)}\left(f^{* *}(t)-f^{*}(t)\right)\right\|_{Y}<\infty\right\}
$$

where $f^{* *}(t)=P f^{*}(t)$. Note that these spaces are not necessarily linear and, in particular, $\|\cdot\|_{Y(\infty, s, X)}$ is not necessarily a norm.

Given a r.i. space $X$ we shall say that $Y$ satisfies the $Q(s, X)$-condition if there exists a constant $C>0$ such that

$$
\left\|t^{-s} \frac{\phi_{X}(t)}{\phi_{Y}(t)} Q f(t)\right\|_{Y} \leq C\left\|t^{-s} \frac{\phi_{X}(t)}{\phi_{Y}(t)} f(t)\right\|_{Y} .
$$

Note that if $b<s$, then we have

$$
t^{-b} \int_{t}^{\infty} f(x) \frac{d x}{x} \leq t^{-s} \int_{t}^{\infty} x^{s-b} f(x) \frac{d x}{x} .
$$

Thus, if $Y$ satisfies the $Q(s, X)$-condition for some $s$, then $Y$ also satisfies the $Q(b, X)$-condition for every $b<s$.

The following lemmas will be useful in what follows. A consequence of our first lemma is that if $Y$ satisfies the $Q(s, X)$-condition, then $Y(\infty, s, X)$ is a Banach space.

Lemma 1. Let $X, Y$ be two r.i. spaces. If $Y$ satisfies the $Q(s, X)$-condition, then for all $f^{* *}(\infty)=0$,

$$
\|f\|_{Y(\infty, s, X)} \simeq\left\|t^{-s} \frac{\phi_{X}(t)}{\phi_{Y}(t)} f^{* *}(t)\right\|_{Y},
$$

with constants of equivalence independent of $f$.

Proof. Obviously,

$$
\left\|t^{-s} \frac{\phi_{X}(t)}{\phi_{Y}(t)}\left(f^{* *}(t)-f^{*}(t)\right)\right\|_{Y} \leq\left\|t^{-s} \frac{\phi_{X}(t)}{\phi_{Y}(t)} f^{* *}(t)\right\|_{Y} .
$$

Conversely, from $\frac{d}{d t} f^{* *}(t)=\frac{f^{*}(t)-f^{* *}(t)}{t}$ and the Fundamental Theorem of Calculus, we have

$$
f^{* *}(t)=\int_{t}^{\infty}\left(f^{* *}(s)-f^{*}(s)\right) \frac{d s}{s}=Q\left(f^{* *}-f^{*}\right)(t),
$$

and the result follows by the $Q(s, X)$-condition.

The next result gives a useful criterion to check the validity of a $Q(s, X)$ condition. 
Lemma 2. Let $X, Y$ be two r.i. spaces. Suppose that

$$
Q(s, X ; Y)=\int_{1}^{\infty} t^{s} h_{Y}\left(\frac{1}{t}\right) M_{Y}(t) M_{X}\left(\frac{1}{t}\right) \frac{d t}{t}<\infty .
$$

Then $Y$ satisfies the $Q(s, X)$-condition.

Proof. We have

$$
\begin{aligned}
t^{-s} \frac{\phi_{X}(t)}{\phi_{Y}(t)} Q f(t) & =\int_{t}^{\infty} t^{-s} \frac{\phi_{X}(t)}{\phi_{Y}(t)} f(x) \frac{d x}{x}=\int_{1}^{\infty} t^{-s} \frac{\phi_{X}(t)}{\phi_{Y}(t)} f(t x) \frac{d x}{x} \\
& \leq \int_{1}^{\infty}(x t)^{-s} f(t x) \frac{\phi_{X}(x t)}{\phi_{Y}(x t)} \sup _{t>0} \frac{\phi_{X}(t)}{\phi_{X}(x t)} \sup _{t>0} \frac{\phi_{Y}(x t)}{\phi_{Y}(t)} x^{s-1} d x \\
& =\int_{1}^{\infty}(x t)^{-s} f(t x) \frac{\phi_{X}(x t)}{\phi_{Y}(x t)} M_{X}(1 / x) M_{Y}(x) x^{s-1} d x .
\end{aligned}
$$

Applying Minkowski's inequality we obtain

$$
\begin{aligned}
\left\|t^{-s} \frac{\phi_{X}(t)}{\phi_{Y}(t)} Q f(t)\right\|_{Y} & \leq \int_{1}^{\infty}\left\|(x t)^{-s} f(t x) \frac{\phi_{X}(x t)}{\phi_{Y}(x t)}\right\|_{Y} M_{X}(1 / x) M_{Y}(x) x^{s-1} d x \\
& \leq \int_{1}^{\infty} h_{Y}(1 / x) M_{X}(1 / x) M_{Y}(x) x^{s-1} d x\left\|t^{-s} f(t) \frac{\phi_{X}(t)}{\phi_{Y}(t)}\right\|_{Y} .
\end{aligned}
$$

Remark 1. In terms of indices, it is easy to see that (2.2) is equivalent to the inequality

$$
s<\underline{\alpha}_{Y}-\bar{\beta}_{Y}+\underline{\beta}_{X} .
$$

Moreover, if $\phi_{X}(t) / \phi_{Y}(t)$ is equivalent to an increasing function, then starting from

$$
t^{-s} \frac{\phi_{X}(t)}{\phi_{Y}(t)} Q f(t) \leq c \int_{t}^{\infty} t^{-s} \frac{\phi_{X}(x)}{\phi_{Y}(x)} f(x) \frac{d x}{x}
$$

and following the same steps as in the proof of the previous lemma we see that if

$$
Q(s, X ; Y)=\int_{1}^{\infty} t^{s} h_{Y}\left(\frac{1}{t}\right) \frac{d t}{t}<\infty,
$$

then $Y$ satisfies the $Q(s, X)$-condition.

\section{Symmetrization inequalities For MOduli OF CONTINUity}

Our first result is a general version of the Pólya-Szegö principle (1.6) valid for r.i. spaces.

Theorem 1. Let $X=X\left(\mathbb{R}^{n}\right)$ be a r.i. space. Then there exists an absolute constant $c>0$ such that for all $f \in X$,

$$
\omega_{X}\left(t, f^{\circ}\right) \leq c \omega_{X}(t, f) .
$$

Proof. We use the interpolation argument given in [22] where the case $n=1$ is proved. We shall use (1.3) and the following auxiliary results, which will be proved below:

$$
\left\|f^{\circ}-g^{\circ}\right\|_{X} \leq\|f-g\|_{X}, \quad f, g \in X
$$


and

$$
\begin{aligned}
K\left(t, f ; X, \stackrel{\circ}{W_{X}^{1}}\right) & =\inf _{\substack{\circ \\
g \in W_{X}^{1}}}\left\{\|f-g\|_{X}+t\|\nabla g\|_{X}\right\} \\
& =\inf _{g \in C_{0}^{\infty}\left(\mathbb{R}^{n}\right)}\left\{\|f-g\|_{X}+t\|\nabla g\|_{X}\right\} .
\end{aligned}
$$

Assuming that (3.2) and (3.3) hold, we can write

$$
\begin{aligned}
K\left(t, f^{\circ} ; X, W_{X}^{1}\right) & \left.=\inf _{g \in C_{0}^{\infty}\left(\mathbb{R}^{n}\right)}\left\{\left\|f^{\circ}-g\right\|_{X}+t\|\nabla g\|_{X}\right\} \text { (by (3.3) }\right) \\
& \leq \inf _{g \in C_{0}^{\infty}\left(\mathbb{R}^{n}\right)}\left\{\left\|f^{\circ}-g^{\circ}\right\|_{X}+t\left\|\nabla g^{\circ}\right\|_{X}\right\} \\
& \left.\leq \inf _{g \in C_{0}^{\infty}\left(\mathbb{R}^{n}\right)}\left\{\|f-g\|_{X}+t\|\nabla g\|_{X}\right\} \text { (by (3.2) and (1.3) }\right) \\
& =K\left(t, f ; X, W_{X}^{1}\right)(\text { by (3.3) }) .
\end{aligned}
$$

Combining the last inequality with the well-known fact that (cf. [14, 4])

$$
K\left(t, g ; X, \stackrel{\circ}{W_{X}^{1}}\right) \simeq \omega_{X}(t, g)
$$

concludes the proof of (3.1) modulo (3.2) and (3.3). To see (3.2) recall that in [18, Theorem 3.5], it is shown that for any convex function $J: \mathbb{R} \rightarrow \mathbb{R}$ such that $J(0)=0$, we have

$$
\int_{\mathbb{R}^{n}} J\left(f^{\circ}(x)-g^{\circ}(x)\right) d x \leq \int_{\mathbb{R}^{n}} J(f(x)-g(x)) d x,
$$

for all $f, g$ positive. We apply this result with $J_{\lambda}(t)=[|t|-\lambda]^{+}$, where $\lambda>0$ is fixed and $[x]^{+}=\max (x, 0)$. It follows that for all $\lambda>0$,

$$
\int_{R^{n}}\left[\left|f^{\circ}(x)-g^{\circ}(x)\right|-\lambda\right]^{+} d x \leq \int_{R^{n}}[|f(x)-g(x)|-\lambda]^{+} d x .
$$

But on account of [4, Exercise 1, page 87], we can write

$$
\left|f^{\circ}-g^{\circ}\right|^{* *}(t)=\inf _{\lambda>0}\left\{\lambda+\frac{1}{t} \int_{\mathbb{R}^{n}}\left[\left|f^{\circ}(x)-g^{\circ}(x)\right|-\lambda\right]^{+} d x\right\} .
$$

Combining (3.5) and (3.4) we get

$$
\left|f^{\circ}-g^{\circ}\right|^{* *}(t) \leq|f-g|^{* *}(t),
$$

and (3.2) follows.

To prove (3.3) we only need to show that for all $f \in X, t>0$,

$$
\inf _{g \in C_{0}^{\infty}\left(\mathbb{R}^{n}\right)}\left\{\|f-g\|_{X}+t\|\nabla g\|_{X}\right\} \leq K\left(t, f ; X, \stackrel{\circ}{W_{X}^{1}}\right) .
$$

Given $\varepsilon>0, t>0$, and $f \in X$, select $g \in \stackrel{\circ}{W_{X}^{1}}$ such that

$$
K\left(t, f ; X, \stackrel{\circ}{W_{X}^{1}}\right) \geq\|f-g\|_{X}+t\|\nabla g\|_{X}-\varepsilon .
$$


In $\stackrel{\circ}{W_{X}^{1}}$ consider the norm $\|f\|_{W_{X}^{1}}=\|f\|_{X}+\|\nabla f\|_{X}$. Then $C_{0}^{\infty}\left(\mathbb{R}^{n}\right)$ is dense in $\left.\stackrel{\circ}{W_{X}^{1}},\|\cdot\|_{W_{X}^{1}}\right)$, and therefore we can select $\left\{g_{n}\right\}_{n \in N} \subset C_{0}^{\infty}\left(\mathbb{R}^{n}\right)$ such that $\left\|g-g_{n}\right\|_{X}$ and $\left\|\nabla g-\nabla g_{n}\right\|_{X}$ both converge to zero as $n \rightarrow \infty$. Using the triangle inequality we see that

$$
\|f-g\|_{X} \geq\left\|f-g_{n}\right\|_{X}-\left\|g-g_{n}\right\|_{X}
$$

and

$$
t\|\nabla g\|_{X} \geq t\left\|\nabla g_{n}\right\|_{X}-t\left\|\nabla g-\nabla g_{n}\right\|_{X}
$$

It follows that

$$
\begin{gathered}
\|f-g\|_{X}+t\|\nabla g\|_{X} \geq\left(\left\|f-g_{n}\right\|_{X}+t\left\|\nabla g_{n}\right\|_{X}\right)-\left\|g-g_{n}\right\|_{X}-t\left\|\nabla g-\nabla g_{n}\right\|_{X} \\
\geq \inf _{g \in C_{0}^{\infty}\left(\mathbb{R}^{n}\right)}\left\{\|f-g\|_{X}+t\|\nabla g\|_{X}\right\} \\
\quad-\left\|g-g_{n}\right\|_{X}-t\left\|\nabla g-\nabla g_{n}\right\|_{X} .
\end{gathered}
$$

Letting $n \rightarrow \infty$ we get

$$
\|f-g\|_{X}+t\|\nabla g\|_{X} \geq \inf _{g \in C_{0}^{\infty}\left(\mathbb{R}^{n}\right)}\left\{\|f-g\|_{X}+t\|\nabla g\|_{X}\right\}
$$

and therefore combining with (3.6) and letting $\varepsilon \rightarrow 0$ we obtain (3.3) concluding the proof of the Theorem.

Remark 2. It is interesting to point out that, although the Pólya-Szegö inequality implies that the nonlinear map $\Theta(f)=f^{\circ}$ is bounded on $\left.\stackrel{\circ}{W_{X}^{1}} \underset{{ }_{\circ}}{\left(\mathbb{R}^{n}\right.}\right)$, Almgren-Lieb 1] proved that the map $\Theta$ is not a continuous operator on $\stackrel{\circ}{W_{X}^{1}}\left(\mathbb{R}^{n}\right)$ if $n \geq 2$. Previously it had been known (cf. Coron [6]) that in one dimension $\Theta$ is continuous on $W_{p}^{1}(\mathbb{R})$. In their paper Almgren-Lieb [1] also show that the map $\Theta$ is not only bounded but also continuous on the Besov spaces $B_{p, p}^{\alpha}\left(\mathbb{R}^{n}\right), 0<\alpha<1$.

Remark 3. The method of proof of Theorem 1 also applies for the general class of rearrangements discussed in [28].

The main tool in our analysis of embeddings of Besov spaces is the following result.

Theorem 2. Let $X=X(\mathbb{R})$ be a r.i. space. Then there exists a constant $c=$ $c(n)>0$ such that for all $t>0$,

$$
f_{0}^{*}(t)=f^{* *}(t)-f^{*}(t) \leq c \frac{\omega_{X}\left(t^{1 / n}, f\right)}{\phi_{X}(t)} .
$$

Proof. Let $\lambda_{f}=$ the distribution function of $f$. Then, as is well known and easy to see,

$$
t f_{o}^{*}(t)=\int_{0}^{t}\left(f^{*}(s)-f^{*}(t)\right) d t=\int_{f^{*}(t)}^{\infty} \lambda_{f}(s) d s
$$


More generally for all $y>0$ we have 8

$$
\int_{\left\{f^{\circ}>y\right\}} f^{\circ}(x) d x=y \lambda_{f} \circ(y)+\int_{y}^{\infty} \lambda_{f}(s) d s .
$$

Inserting $y=f^{*}(t)$ in 3.9 wet

$$
\begin{aligned}
\int_{\left\{f^{\circ}>f^{*}(t)\right\}} f^{\circ}(x) d x & =f^{*}(t) \lambda_{f^{\circ}}\left(f^{*}(t)\right)+\int_{f^{*}(t)}^{\infty} \lambda_{f^{\circ}}(s) d s \\
& =f^{*}(t) \int_{\left\{f^{\circ}>f^{*}(t)\right\}} d x+\int_{f^{*}(t)}^{\infty} \lambda_{f^{\circ}}(s) d s .
\end{aligned}
$$

Therefore

$$
\begin{aligned}
\int_{\left\{f^{\circ}>f^{*}(t)\right\}}\left(f^{\circ}(x)-f^{*}(t)\right) d x & =\int_{f^{*}(t)}^{\infty} \lambda_{f^{\circ}}(s) d s \\
& =\int_{f^{*}(t)}^{\infty} \lambda_{f}(s) d s\left(\text { since } \lambda_{f^{\circ}}=\lambda_{f}\right) \\
& =t f_{o}^{*}(t)(\text { by }(\underline{3.8})) .
\end{aligned}
$$

It will be convenient to introduce some notation. Let $A=\left\{x \in \mathbb{R}^{n}: f^{\circ}(x)>f^{*}(t)\right\}$, and let $B(x, h)=$ ball with center $x$ and radius $h$. Then

$$
|A|=\lambda_{f} \circ\left(f^{*}(t)\right)=\lambda_{f}\left(f^{*}(t)\right) \leq t .
$$

Pick $h=\left(\frac{2 t}{c_{n}}\right)^{1 / n}$ and for each $x \in A$ let

$$
A_{x}=\left\{y \in B(x, h): f^{\circ}(y) \leq f^{*}(t)\right\}
$$

Since

$$
\begin{aligned}
2 t & =|B(x, h)|=\left|A_{x}\right|+\left|\left\{y \in B(x, h): f^{\circ}(y)>f^{*}(t)\right\}\right| \\
& \leq\left|A_{x}\right|+|A| \leq\left|A_{x}\right|+t
\end{aligned}
$$

we get that

$$
\left|A_{x}\right| \geq t
$$

8

$$
\begin{array}{r}
\int_{\left\{f^{\circ}>y\right\}} f^{\circ}(x) d x=\int_{0}^{\infty}\left(f^{\circ}(x)-y\right)^{+} d x+y \lambda_{f^{\circ}}(y)=\int_{0}^{\infty} \lambda_{\left(f^{\circ}-y\right)}(s) d s+y \lambda_{f} \circ(y) \\
=\int_{y}^{\infty} \lambda_{f}(s) d s+y \lambda_{f} \circ(y) .
\end{array}
$$


Rewriting (3.10) using this notation we have

$$
\begin{aligned}
t f_{o}^{*}(t) & =\int_{A}\left(f^{\circ}(x)-f^{*}(t)\right) d x \\
& \leq \int_{A}\left(f^{\circ}(x)-\frac{1}{\left|A_{x}\right|} \int_{A_{x}} f^{\circ}(y) d y\right) d x \\
& =\int_{A}\left(\frac{1}{\left|A_{x}\right|} \int_{A_{x}}\left(f^{\circ}(x)-f^{\circ}(y)\right) d y\right) d x \\
& \leq \frac{1}{t} \int_{A} \int_{A_{x}}\left(f^{\circ}(x)-f^{\circ}(y)\right) d y d x \quad(\text { by (3.11) }) \\
& \leq \frac{1}{t} \int_{A}\left(\int_{B(x, h)}\left|f^{\circ}(x)-f^{\circ}(y)\right| d y\right) d x \\
& =\frac{1}{t} \int_{A}\left(\int_{B(0, h)}\left|f^{\circ}(x)-f^{\circ}(x+u)\right| d u\right) d x \\
& =\frac{1}{t} \int_{B(0, h)}\left(\int_{A}\left|f^{\circ}(x)-f^{\circ}(x+u)\right| d x\right) d u \\
& \leq \frac{1}{t} \int_{B(0, h)}\left\|f^{\circ}(\cdot)-f^{\circ}(\cdot+u)\right\|_{X} \phi_{X^{\prime}}(|A|) d u \quad(\text { Hölder's inequality }) \\
& \leq \frac{\phi_{X^{\prime}}(t)}{t} \int_{B(0, h)} \omega_{X}\left(h, f^{\circ}\right) d u \\
& =\frac{\phi_{X^{\prime}}(t)}{t} \omega_{X}\left(\left(2 t / c_{n}\right)^{1 / n}, f^{\circ}\right)\left(\left(2 t / c_{n}\right)^{1 / n}\right)^{n} \\
& \leq c \phi_{X^{\prime}}(t) \omega_{X}\left(t^{1 / n}, f^{\circ}\right) \quad\left(\text { since } \omega_{X}(\alpha t, f) \leq(1+\alpha) \omega_{X}(t, f)\right) .
\end{aligned}
$$

Applying Theorem 1, and recalling that $\phi_{X^{\prime}}(t) \phi_{X}(t)=t$, we therefore see that,

$$
f_{o}^{*}(t) \leq c \omega_{X}\left(t^{1 / n}, f\right) \frac{\phi_{X^{\prime}}(t)}{t}=c \frac{\omega_{X}\left(t^{1 / n}, f\right)}{\phi_{X}(t)},
$$

concluding the proof.

The integrated version of Theorem 2 extends and simplifies a result stated in 4 , Chapter 5, Theorem 4.19] for $L^{p}$ spaces.

Corollary 1. There exists a constant $c>0$ such that for all $f \in X$, with $f^{*}(\infty)=$ 0 ,

$$
f^{* *}(t) \leq c \int_{t^{1 / n}}^{\infty} \frac{\omega_{X}(s, f)}{\phi_{X}\left(s^{n}\right)} \frac{d s}{s} .
$$

Proof. By the Fundamental Theorem of Calculus,

$$
\begin{aligned}
f^{* *}(t) & =\int_{t}^{\infty}\left(f^{* *}(s)-f^{*}(s)\right) \frac{d s}{s} \\
& \leq c \int_{t}^{\infty} \frac{\omega_{X}\left(s^{1 / n}, f\right)}{\phi_{X}(s)} \frac{d s}{s} \text { (by (3.7)) }
\end{aligned}
$$

and (3.12) follows. 


\section{Embedding theOREms for Besov spaces}

Let $X=X\left(\mathbb{R}^{n}\right)$, let $Y$ be a r.i space over $[0, \infty)$ and let $0<s<1$. We define the Besov space $\stackrel{\circ}{X}_{X, Y}^{s}\left(\mathbb{R}^{n}\right)$ to be the closure of $C_{0}^{\infty}\left(\mathbb{R}^{n}\right)$ under the seminorm

$$
\|f\|_{\dot{\mathrm{B}}_{X, Y}^{s}}=\left\|\frac{t^{-s / n} \omega_{X}\left(f, t^{1 / n}\right)}{\phi_{Y}(t)}\right\|_{Y} .
$$

Remark 4. If in the definition of $\stackrel{\circ}{\mathrm{B}}_{X, Y}^{s}\left(\mathbb{R}^{n}\right)$ we let $X=L^{p}\left(\mathbb{R}^{n}\right)$ and $Y=L^{q}([0, \infty))$, $1 \leq q<\infty$, then $\phi_{Y}(t)=t^{1 / q}$ and we have

$$
\begin{aligned}
\left\|\frac{t^{-s / n} \omega_{L^{p}}\left(t^{1 / n}, f\right)}{\phi_{Y}(t)}\right\|_{Y} & =\left(\int_{0}^{\infty}\left(t^{-s / n} \omega_{L^{p}}\left(t^{1 / n}, f\right)\right)^{q} \frac{d t}{t}\right)^{1 / q} \\
& \simeq\left(\int_{0}^{\infty}\left(t^{-s} \omega_{L^{p}}(t, f)\right)^{q} \frac{d t}{t}\right)^{1 / q} .
\end{aligned}
$$

Thus, $f \in \stackrel{\circ}{\mathrm{B}}_{L^{p}, L^{q}}\left(\mathbb{R}^{n}\right)$ if and only if $f$ belongs to the classical Besov space $\stackrel{\circ}{\mathrm{B}}_{p, q}^{s}\left(\mathbb{R}^{n}\right)$. The usual modifications apply when $q=\infty$.

From Theorem 2 we immediately get

Corollary 2. Let $X, Y$ be r.i. spaces, $0<s<1$. Then

$$
\stackrel{\text { ss }}{B_{X, Y}}\left(\mathbb{R}^{n}\right) \subset Y\left(\infty, \frac{s}{n}, X\right)\left(\mathbb{R}^{n}\right) .
$$

Proof. By Theorem 2,

$$
\|f\|_{Y\left(\infty, \frac{s}{n}, X\right)} \leq c\left\|\frac{t^{-s / n} \omega_{X}\left(f, t^{1 / n}\right)}{\phi_{Y}(t)}\right\|_{Y} .
$$

Remark 5. The extension to higher order $(s \geq 1)$ Besov spaces, as well as the embeddings of Besov spaces on domains, will be considered in a forthcoming paper [19.

4.1. Some concrete examples. Suppose that $Y=L_{q}\left(\mathbb{R}^{n}\right)$. Then we can give a more precise version of Lemma 1 (see also [23]).

Lemma 3. Let $X$ be a r.i space. Let $s \in(-1,1)$ and suppose $q \geq 1$. Let $v(t)=$ $t^{-s-1 / q} \phi_{X}(t)$. Then the following statements are equivalent:

i) $s<\underline{\beta}_{X}$

ii) $L_{q}\left(\overline{\mathbb{R}}^{n}\right)$ satisfies the $Q(s, X)$-condition;

iii) if $f^{*}(\infty)=0$,

$$
\|f\|_{L^{q}(\infty, X)}=\left\|v(t)\left(f^{* *}(t)-f^{*}(t)\right)\right\|_{q} \simeq\left\|v(t) f^{* *}(t)\right\|_{q} .
$$

Proof. $i) \rightarrow$ ii) An easy computation shows that condition (2.2) holds and then Lemma 2 applies. ii) $\rightarrow$ iii) Follows from Lemma 1. To conclude the proof we show $i i i) \rightarrow i$ ): By Fubini we readily see that (we need $f^{*}(\infty)=0$, otherwise $Q f^{*}$ does not exist)

$$
Q f^{* *}=Q \circ P f^{*}=Q f^{*}+P f^{*}=P \circ Q f^{*}=\left(Q f^{*}\right)^{* *} .
$$

Thus

$$
\left(Q f^{*}\right)^{* *}(t)-Q f^{*}(t)=P f^{*}(t)=f^{* *}(t)
$$


Consider now the r.i. space $H$ defined by the norm

$$
\|h\|_{H}=\left\|v(t) f^{* *}(t)\right\|_{q} .
$$

Then, by condition iii),

$$
\begin{aligned}
\phi_{H}(r) & =\left\|\chi_{[0, r]}\right\|_{H} \simeq\left\|v(t)\left(\chi_{[0, r]}^{* *}(t)-\chi_{[0, r]}(t)\right)\right\|_{q} \\
& =r\left(\int_{r}^{\infty} v(t)^{q} \frac{d t}{t^{q}}\right)^{1 / q} .
\end{aligned}
$$

On the other hand, since $\phi_{X}$ is increasing,

$$
\int_{r}^{\infty} v(t)^{q} \frac{d t}{t^{q}}=\int_{r}^{\infty} t^{-s q-1} \phi_{X}(t)^{q} \frac{d t}{t^{q}} \geq \frac{\phi_{X}(r)^{q} r^{-s q-q}}{q(s-1)} .
$$

Similarly

$$
\begin{aligned}
\phi_{X}(r)^{q} r^{-s q-q} & \preceq \phi_{X}(r)^{q} r^{-s q-q} \int_{r}^{2 r} \frac{d t}{t} \preceq \int_{r}^{2 r} \phi_{X}(t)^{q} t^{-s q-q} \frac{d t}{t} \\
& \leq \int_{r}^{\infty} t^{-s q-1} \phi_{X}(t)^{q} \frac{d t}{t^{q}}=\int_{r}^{\infty} v(t)^{q} \frac{d t}{t^{q}}
\end{aligned}
$$

Thus

$$
\phi_{H}(r) \simeq \phi_{X}(r) r^{-s}
$$

and

Finally, since

$$
\underline{\beta}_{H}=-s+\underline{\beta}_{X} .
$$

$$
\begin{aligned}
\|f\|_{H} & =\left\|v(t)\left(\left(Q f^{*}\right)^{* *}(t)-Q f^{*}(t)\right)\right\|_{q} \quad(\text { by }(4.1)) \\
& \left.\simeq\left\|v(t)\left(Q f^{*}\right)^{* *}(t)\right\|_{q} \quad(\text { by condition } i i i)\right) \\
& =\left\|Q f^{*}\right\|_{H}
\end{aligned}
$$

it follows that $Q: H \rightarrow H$ is bounded, which implies that $\underline{\beta}_{H}>0$.

\section{ACKNOWLEDGEMENT}

We are grateful to the referee for useful remarks and suggestions to improve the presentation of the paper.

\section{REFERENCES}

[1] F. J. Almgren and E. H. Lieb, Symmetric decreasing rearrangement is sometimes continuous, J. Amer. Math. Soc. 2 (1989), 683-773. MR.1002633 (90f:49038)

[2] J. Bastero, M. Milman and F. Ruiz, A note in $L(\infty, q)$ spaces and Sobolev embeddings, Indiana Univ. Math. J. 52 (2003), 1215-1230. MR2010324 (2004h:46025)

[3] C. Bennett, R. De Vore and R. Sharpley, Weak- $L^{\infty}$ and BMO, Ann. of Math. 113 (1981), 601-611. MR:0621018 (82h:46047)

[4] C. Bennett and R. Sharpley, Interpolation of Operators, Academic Press, Boston (1988). MR0928802 (89e:46001)

[5] H. Brézis and S. Wainger, A note on limiting cases of Sobolev embeddings and convolution inequalities, Comm. Partial Diff. Eq. (1980), 773-789. MR0579997 (81k:46028)

[6] J.-M. Coron, The continuity of rearrangement in $W^{1, p}(\mathbb{R})$, Ann. Scuola Norm. Sup. Pisa Cl. Sci. 11 (1984), 57-85. MR0752580 (86a:46035)

[7] M. Cwikel and E. Pustylnik, Sobolev type embeddings in the limiting case, J. Fourier Anal. Appl. 4 (1998), 433-446. MR1658620(99k:46055) 
[8] J. J. F. Fournier, Mixed norms and rearrangements: Sobolev's inequalities and Littlewood's inequality, Ann. Math. Pura Appl. 148 (1987), 51-76. MR0932758(89e:46037)

[9] A. Garsia, Combinatorial inequalities and smoothness of functions, Bull. Amer. Math. Soc. 82 (1976), 157-170. MR0582776 (58:28362)

[10] A. Garsia and E. Rodemich, Monotonicity of certain functionals under rearrangements, Ann. Inst. Fourier (Grenoble) 24 (1974), 67-116. MR0414802 (54:2894)

[11] K. Hansson, Imbedding theorems of Sobolev type in potential theory, Math. Scand. 45 (1979), 77-102. MR0567435 (81j:31007)

[12] P. Hajlasz, Sobolev inequalities, truncation method and John domains, in Papers on Analysis: a volume dedicated to Olli Martio, Report. Univ. Jyväskylä 83 (2001), 109-126. MR.1886617 (2003a:46052)

[13] C. Herz, Lipschitz spaces and Bernstein's theorem on absolutely convergent Fourier transforms, J. Math. Mech. 18 (1968), 283-323. MR0438109 (55:11028)

[14] H. Johnen and K. Scherer, On the equivalence of the $K$-functional and moduli of continuity and some applications, in Constructive theory of functions of several variables, pp. 119-140, Lecture Notes in Math. 571, Springer, Berlin, 1977. MR0487423 (58:7060)

[15] V. S. Klimov, Imbedding theorems for symmetric spaces, Math. USSR -Sbornik 8 (1969), 161-168. MR0511930 (58:23544)

[16] V. I. Kolyada, Estimates of rearrangements and embedding theorems, Mat. Sb. 136 (1989), 3-29. MR0945897 (89h:46049)

[17] V. I. Kolyada, Rearrangements of functions, and embedding theorems, Russian Math. Surveys 44 (1989), 73-117. MR.1040269 (91i:46029)

[18] E. H. Lieb and M. Loss, Analysis, American Mathematical Society, Providence, RI, 2001. MR.1817225 (2001i:00001)

[19] J. Martín and M. Milman, Higher order symmetrization inequalities and applications (preprint).

[20] V. G. Maz'ya, Sobolev spaces, Springer-Verlag, New York, 1985. MR0817985 (87g:46056)

[21] V. G. Maz'ya, On certain integral inequalities for functions of many variables. Problems of Mathematical Analysis, Leningrad Univ. 3 (1972), 33-68 (Russian). English trans. J. Soviet Math. 1 (1973), 205-234.

[22] M. Milman, On interpolation of entropy and block spaces, Quart. J. Math. (2) 45 (1994), 531-540. MR1315462 (96b:46107)

[23] M. Milman and E. Pustylnik, On sharp higher order Sobolev embeddings, Comm. Cont. Math. 6 (2004), 1-17. MR.2068850 (2005f:46068)

[24] J. Mossino, Inégalités isopérimértriques et applications en physique, Travaux en Cours, Hermann, Paris, 1984. MR0733257 (85k:49002)

[25] J. Peetre, Espaces d'interpolation et théorème de Sobolev, Ann. Inst. Fourier (Grenoble) 16 (1966), 279-317. MR0221282 (36:4334)

[26] E. Pustylnik, Sobolev type inequalities in ultrasymmetric spaces with applications to OrliczSobolev embeddings, Journal of Function Spaces and Applications 3 (2005), 183-208. MR2135650 (2006a:46040)

[27] E. Stein, Singular integrals and differentiability properties of functions, Princeton University Press, New Jersey, 1970. MR0290095 (44:7280)

[28] J. Ván Schaftingen, Universal approximation of symmetrizations by polarizations, Proc. Amer. Math. Soc. 134 (2006), 177-186. MR2170557

Department de Matemàtiques, Universitat Autónoma de Barcelona, 08193 BellaTERRA (BARCELONA) SPAin

E-mail address: jmartin@mat.uab.es

Department of Mathematics, Florida Atlantic University, Boca Raton, Florida 33431

E-mail address: interpol@bellsouth.net 